\title{
Les Utopies adolescentes de Robert Louis Stevenson
}

\section{Cyril Besson}

\section{(2) OpenEdition}

\section{Journals}

\section{Édition électronique}

URL : http://journals.openedition.org/etudesecossaises/71

DOI : 10.4000/etudesecossaises.71

ISSN : 1969-6337

\section{Éditeur}

UGA Éditions/Université Grenoble Alpes

\section{Édition imprimée}

Date de publication : 30 janvier 2008

Pagination : 69-78

ISBN : 978-2-84310-110-6

ISSN : 1240-1439

\section{Référence électronique}

Cyril Besson, «Les Utopies adolescentes de Robert Louis Stevenson », Études écossaises [En ligne], 11 | 2008, mis en ligne le 30 janvier 2009, consulté le 08 septembre 2020. URL : http://

journals.openedition.org/etudesecossaises/71 ; DOI : https://doi.org/10.4000/etudesecossaises.71

Ce document a été généré automatiquement le 8 septembre 2020

(c) Études écossaises 


\title{
Les Utopies adolescentes de Robert Louis Stevenson
}

\author{
Cyril Besson
}

1 Les œuvres de Robert Louis Stevenson seraient-elles victimes d'un malentendu? Une méprise persistante consiste en effet à considérer l'Écossais comme un simple auteur de récits d'aventures pour enfants, là où ce thème n'occupe finalement qu'une partie de son œuvre. Mais l'on pourrait dire que même cette section du travail de Stevenson n'a pas nécessairement eu les faveurs de la critique, relevant d'un genre quelque peu méprisé, et connue surtout, en France, à travers L'île au Trésor. Jean-Pierre Naugrette rappelle pourtant qu'il existe un autre pan de cette partie de l'œuvre ${ }^{1}$, notamment un diptyque écossais constitué de Kidnapped ${ }^{2}$ et de sa suite Catriona ${ }^{3}$. Ces deux romans racontent, sur le mode homodiégétique, les aventures du jeune David Balfour. Dans un premier temps, il nous détaille sa longue lutte, couronnée d'un succès tout partiel à la fin de Kidnapped, pour recouvrer son héritage dans un monde encore marqué par les séquelles des troubles de 1745 (ses aventures se déroulent en 1751) puis, dans le second opus, expose sa rencontre avec sa bien aimée, le personnage-titre, et son accession à ses droits après maintes péripéties.

Or le récit d'aventures chez Stevenson ne relève pas d'un simple désir d'évasion, cet escapism de petit garçon qui lui est beaucoup reproché. Sans aller peut-être jusqu'aux extrêmes d'Alan Sandison, qui voit Stevenson comme le premier moderniste dans un ouvrage pourtant souvent pénétrant ${ }^{4}$, on peut lui reconnaître de nombreuses qualités, dont une fascination pour un jeu formel où rien n'est ce qu'il semble être, et surtout une volonté foncière d'originalité. Le récit d'aventures chez Stevenson, loin d'être un simple abâtardissement du Bildungsroman, est surtout l'occasion de s'émanciper des codes pré-établis pour fonder un domaine singulier, bien à soi, que nous qualifierons d'utopique dans le sens où il impose un positionnement très particulier, en retrait du monde comme nous tenterons de le démontrer, qui rejaillit sur le personnage principal autant que sur la narration elle-même. Avant cette étape ultime, il aura fallu se débarrasser des figures paternelles qui hantent l'œuvre, mais jamais autant que dans le diptyque écossais, et prendre leur place, pour renverser un ordre injuste et aliénant, 
puis fonder son ordre à soi, cette utopie adolescente d'un monde débarrassé de toute autorité autre que la sienne propre.

Il s'agira dans un premier temps de chercher à dissiper une méprise insistante qui consiste à envisager Kidnapped et Catriona comme des Bildungsromans. Pour démontrer qu'ils ne rentrent pas dans cette catégorie, nous commencerons par en poser les bases, et prendrons comme caractéristiques du Bildungsroman celles qu'identifie Jerome Hamilton Buckley dans son ouvrage Season of Youth ${ }^{5}$, qui offre l'avantage de fournir une synthèse opératoire d'un genre extrêmement varié. Pour aller à l'essentiel, selon Buckley, le Bildungsroman, auquel il préfère donner le nom de "roman de formation " (apprenticeship novel), dans sa forme anglo-saxonne, s'articule autour de récurrences :

A child of some sensitivity grows up in the country or in a provincial town, where he finds constraints, social and intellectual, placed upon the free imagination. His family, especially his father, proves doggedly hostile to his creative instincts or flights of fancy, antagonistic to his ambitions, and quite impervious to the new ideas he has gained from unprescribed reading. His first schooling, even if not totally inadequate, may be frustrating insofar as it may suggest options not yet available to him. He therefore, sometimes at a quite early age, leaves the repressive atmosphere of home (and also the relative innocence), to make his way independently in the city [...]. There his "real" education begins, not only his preparation for a career but also - and often more importantly-his direct experience of urban life. The latter involves at least two love affairs or sexual encounters, one debasing, one exalting, and demands that in this respect and others the hero reappraise his values. By the time he has decided, after painful soul-searching, the sort of accommodation to the modern world he can honestly make, he has left his adolescence behind and entered upon his maturity. His initiation complete, he may then visit his old home, to demonstrate by his presence the degree of his success or the wisdom of his choice ${ }^{6}$.

Les italiques dans la citation ci-dessus sont de ma part, et soulignent les points de convergence, souvent relatifs et partiels, avec l'itinéraire de David, pris dans sa globalité ; il apparaît d'emblée que le Bildungsroman a chez Stevenson une saveur bien différente de celle des grands romans de Dickens. Certes, le jeune monsieur Balfour, orphelin dès le début de Kidnapped, aspire effectivement à plus que la vie à la campagne, et part rejoindre non pas la ville, mais l'ancien domaine familial (ou ce qu'il en reste), pour y retrouver son unique "parent", son oncle Ebenezer (frère du père, dont il représente d'un certain point de vue une survivance en double maléfique fantasmé), qui se conforme à ce que Buckley dit un peu plus loin des pères ou substituts paternels dans le Bildungsroman :

The growing child [...] more often than not will be orphaned or at least fatherless [...]. But if not deprived by death of a father, who presumably would have been a true guide and protector, he will almost certainly be repelled [...] by a living father who mistrusts and seeks to thwart his strongest drives and fondest desires. [...]. The loss of the father, either by death or alienation, usually symbolizes or parallels a loss of faith in the values of the hero's home and family and leads inevitably to the search for a substitute parent or creed [...]. The defection of the father becomes accordingly the principal motive force in the assertion of the youth's independence. [...] [He] must make his own way resolutely through the forests of experience ${ }^{7}$. son frère aîné, qu'il incarne une dégénérescence de la lignée, ce dont témoigne son goût immodéré pour le seul porridge accompagné de petite bière - symboles de sa décrépitude sénile. La malignité rabougrie d'Ebenezer dans Kidnapped forcera David à lui livrer une lutte sans merci, source pour le jeune héros d'une nouvelle appréhension du monde, pleine de désillusions, prélude à sa ré-appropriation de son héritage. Le 
conflit familial a pour but ultime de revitaliser la Maison de Shaws, la dynastie des Balfour en quelque sorte, par la jeunesse, l'appétit et la solide santé de son dernier représentant - mais il s'agit bien d'une visée ultime, puisque Kidnapped s'arrête alors que l'oncle, pourtant déjà dépouillé du peu de vigueur qui lui restait, est encore vivant, et la propriété de David encore mal établie. Son éducation se poursuit dans Catriona, où il lui faut dans un premier temps éprouver les vicissitudes de la ville d'Édimbourg. Entre autres péripéties, il y fait la rencontre, d'une part, du personnage-titre, incarnation de la pureté saine des Highlands qui ramène David à un certain sens du naturel et, d'autre part, de Miss Barbara Grant, exacte antithèse de la première sans jamais véritablement être sa rivale dans le jeu ambigu auquel elle se livre avec le jeune homme. Son influence n'en est pourtant pas moins corruptrice, puisqu'elle menace souvent de faire basculer David dans une urbanité qui ne lui sied guère. Après moult péripéties, il revient bien (fort brièvement) au lieu des origines, la maison de Shaws, même s'il se contente de l'observer de loin, alors que son oncle s'y meurt; mort d'ailleurs consommée quelques pages plus loin, mais déjà effective dans le texte, tant tout ce qui touche à Ebenezer est présenté de manière laconique dans Catriona. Ce n'est cependant pas, loin s'en faut, la fin des aventures de David.

6 On le voit, ce squelette narratif correspond très grossièrement au schéma du Bildungsroman selon Buckley, mais il s'agit pour Stevenson de l'intégrer partiellement pour le dépasser. Car il est une différence fondamentale entre le modèle établi par Buckley et le point de départ chez l'Écossais : la première étape, celle de la petite enfance et de la croissance du personnage principal, ne nous est pas donnée, car "l'enfant", à dix-huit ans, se présente à nous en grande partie déjà formé. Cette particularité donne toute son originalité au narrateur "adolescent» stevensonien, dont la clef est que, d'un bout à l'autre du diptyque, il n'évoluera pas. L'épisode avec M. Campbell, au tout début de Kidnapped, donne assez bien à voir qu'à peine lancé sur sa quête, David se défait déjà des conventions et des cadres. Le pasteur fait à David trois cadeaux, qui s'énoncent comme autant de formes géométriques (l'un sphérique, le second plat et le dernier cubique). Le lecteur féru de contes, qui se prépare à l'analyse minutieuse de l'usage, judicieux ou non, qui sera fait des objets visant à aider le héros sur le chemin de la découverte de la Vie et de la quête de son identité, ne manquera pas d'être déçu de voir comment, leur trivialité confondante révélée au second chapitre, David s'en débarrasse sans plus jamais y repenser. L'amateur de mythologie, lui, constate que ces trois objets mystérieux forment presque une énigme, à laquelle il manque cependant la formulation explicite d'une question; qu'est donc mon tout? M. Campbell ne détient donc pas plus les réponses à une question qu'il ne sait pas poser, qu'il n'attribue les outils pour affronter la vie; piètre sphinx, il n'est pas adjuvant plus efficace - et il incarne la plus acceptable des figures paternelles dans les deux romans. Les modèles classiques cèdent le pas au non-conformisme de Stevenson, et cet incident est le prélude à une destitution systématique, presque acharnée, des figures d'autorité, toutes invalidées comme modèles imparfaits. Face au respect littéral mais astucieux de la loi de Rankeillor, ou aux manigances énigmatiques de Prestongrange, qui tous prétendent agir au mieux de ses intérêts en passant outre son contrôle de la situation, David éprouve surtout une méfiance, qu'il dénoncera certes à plusieurs reprises comme infondée et pleine d'ingratitude, mais pour laquelle il ne s'excusera jamais dans l'espace du roman, ce que Dickens ne manque pas d'imposer à Pip, par exemple. On ressort du diptyque écossais avec le sentiment que le respect de David pour les figures d'autorité n'est que de pure façade; elles sont nombreuses, en 
effet, et pour tout dire presque systématiques, ces scènes d'échanges fort vifs avec un Rankeillor, un Prestongrange ou un James More. Mais nul, à part peut-être Alan, n'aura droit à la présentation contrite des excuses sincères du jeune homme, pourtant figure imposée du récit d'adolescence et de formation, articulée sur sa dimension confessionnelle. Et s'il fallait encore s'en persuader, l'absence totale de cette dimension confessionnelle achèverait de convaincre que le diptyque n'appartient pas au genre du Bildungsroman. À qui se confesser, en effet, quand on est soi-même seul maitre et possesseur de sa nature, inflexible et intransigeante, quand le refus de la culpabilité et du repentir est érigé en principe existentiel ? Il faudra d'ailleurs s'intéresser plus loin à l'épineux problème du destinataire du texte, bien différent de ce que l'on pourrait croire, comme nous l'apprend une conclusion lapidaire mais pleine de sens. Contentons-nous pour l'instant d'observer que personne ne fera ravaler sa morgue à David, une arrogance revendiquée dont il ne se départit jamais vraiment, et qui exsude à chaque seconde de ces confrontations avec les figures d'autorité.

7 Ainsi donc, David semble déjà avoir renoncé à l'utopie de l'enfance, celle qui consiste à croire qu'il y aura toujours un guide pour vous montrer la voie, afin peut-être d'en fonder une autre, où l'on serait (partiellement) débarrassé de ce joug. Sans doute est-ce là une manière quelque peu cynique de présenter les choses, mais, conformément aux données de sa biographie, tuer le père, voilà le grand projet stevensonnien. Et tuer le père, cela commence par une liquidation partielle de l'héritage de Walter Scott, père fondateur de la littérature écossaise, qui tient presque du lapsus calami. Il est frappant de constater à quel point Stevenson réutilise le schéma que Walter Scott avait établi dans Waverley ${ }^{8}$ : Alan Breck, incarnation de tous les archaïsmes des Highlands, non dénués de noblesse il est vrai, n'est ainsi pas sans rappeler Fergus dans le roman fondateur de Scott, et il y a un peu de Waverley dans le Lowlander David, aux prises avec une Histoire qu'il mettra moins de temps à renier que son illustre modèle. Mais cet écho de Scott se fait plus insistant encore à travers les figures paternelles, dont chacune a un intérêt particulier, mais qui toutes se ressemblent: la parenté est saisissante entre Rankeillor et Prestongrange, l'un occupant de sa stature impérieuse la fin de Kidnapped, l'autre, le début de Catriona, au point qu'ils sont presque interchangeables. Latinistes émérites et pour tout dire un peu pédants, très au fait des usages de la société dans laquelle ils choisissent d'évoluer, coutumiers d'intrigues qui finissent par les dépasser, ils ne sont pas sans faire penser à un Bradwardine (la sensibilité antiquaire et le comique en moins), ou à un Hugh Redgauntlet. Dans des romans obsédés par la récurrence des portes, motif fondamental chez Stevenson, ils incarnent des verrous qu'il s'agit de faire sauter : l'un fait se clore Kidnapped sur un inachèvement et un blocage (l'héritage de David à la fin n'est en fait que de pure forme, comme se chargera de le démontrer le début du second opus) ; l'autre empêche David de prendre toute sa dimension, et le contraint à plusieurs reprises à séjourner dans des pièces fermées, ainsi que d'autres espaces infranchissables, et ne fait à ce titre pas mieux qu'Ebenezer. Ce n'est qu'en dépassant les limites qu'ils imposent, en se jouant d'eux, que David pourra trouver une certaine liberté. Transgresser la loi, et surtout celle édictée par le père, voilà ce que David fait continuellement. Dès lors, Prestongrange peut être considéré comme la figure paternelle la plus menaçante, par la toute-puissance insidieuse qu'il exerce sur ses filles. Les libertés qu'il prend avec elles, notamment lorsqu'il demande à David, devant elles, laquelle la nature a le plus gâté ${ }^{9}$ ou bien encore lorsqu'il en fait ses Cerbère pour retenir « M. Balfour ", se ménageant ainsi un peu de répit pour intriguer en paix, en sont la preuve. Mais c'est surtout sa relation 
ambiguë avec l'aînée, Miss Grant, qui ne lasse pas d'étonner. Véritable Athéna sortie du corps de Zeus, la jeune fille semble entièrement dévouée à la cause de son père, quelle qu'elle soit, et l'usage récurrent qu'elle fait de la formulation «sa seigneurie mon père » (" his Lordship my Papa »), en témoigne assez bien, même si la demoiselle affecte l'ironie. Bien plus que la rivale de Catriona, elle est l'instrument dévoué de son père, l'exact négatif de David, dont elle est le véritable double inversé, là où Alan serait plutôt un prolongement fantasmatique (l'éternel révolté toujours lancé sur quelque aventure). Elle constitue un vrai danger pour l'orphelin, dans la mesure où elle sera la seule à savoir le faire plier, littéralement, quand elle lui impose, sous un faux prétexte que le jeune homme pourtant reconnaît pour faux, de se mettre à genoux devant elle, par caprice plus que pour toute autre raison. Lui qui pourtant ne courbe jamais l'échine s'exécute sans vraiment rechigner, mais sans trop savoir pourquoi. Miss Grant incarne la séduction d'une soumission facile à un pouvoir tutélaire, une démission qui permet de trouver une place assurée dans un carcan déterministe (elle n'a qu'une seule fonction, jouer de son charme pour imposer les oukases paternels), là où David en est réduit à négocier sa place, toujours maladroitement, dans un ordre dans lequel il ne se reconnaît pas.

8 C'est tout l'objet de l'opposition radicale entre Miss Grant et Miss Drummond, entre Barbara et Catriona. Même s'il lui reconnaît d'immenses qualités, David confesse d'emblée un surprenant manque d'attirance envers Barbara ; la raison en est peut-être qu'elle gravite dans la sphère d'attraction de Prestongrange, et qu'il est dès le départ évident qu'il sera impossible de l'en détourner. David ne se préoccupe donc pas d'arracher Miss Grant aux griffes de son père; mais il y a un véritable enjeu pour le jeune homme à pousser Catriona à se détacher du sien, James More. Elle aussi semble, au départ, prête à tous les sacrifices pour lui, allant, pour le faire évader, jusqu'à prendre sa place dans le cachot où il est enfermé. De ce fait, James More est à maintes reprises ouvertement posé par David comme un obstacle dans sa conquête de Catriona, un véritable rival pour son affection, et l'arrivée du Highlander en Hollande le montre assez bien, puisque les deux tourtereaux vivaient jusque-là leur amour de manière presque édénique, et à vrai dire asexuée. Il est incompatible d'aimer à la fois ces deux hommes, et les déchirements de Catriona le font assez bien sentir. Et si le Highlander finit par offrir sa fille, au cours d'une transaction presque commerciale où il a un intérêt évident, c'est au moment exact où les relations entre David et la jeune fille se sont dégradées au point que leur liaison semble terminée avant que d'avoir véritablement commencé. David décline l'offre de mariage, peut-être précisément parce que cette figure paternelle y consent. Pour mériter sa compagne, il s'agit plutôt pour David de voler sa fille à James, de la lui enlever, c'est-à-dire passer du kidnappé au kidnappeur, dans une stratégie oedipienne inconsciente. À ce titre, la destination du texte à un jeune public explique certes en partie le peu d'ardeur charnelle que David manifeste envers sa bien-aimée, mais en partie seulement, et sa tendance à l'affubler du nom si peu doux de «my little friend » finit de convaincre qu'il y a derrière sa séduction de Catriona autre chose qu'une attirance amoureuse véritable. Il y a presque une obstination de Stevenson à pousser la fille du Highlander James More à renier ce père que tous considèrent comme abject, veule et lâche, tout comme à pousser David à renier le sien, symboliquement, chaque fois qu'il lui fait refuser tout lien de parenté avec un médecin allié aux rebelles en 1745, qui portait le patronyme de Balfour, et dont tous veulent savoir s'il est son père. Que tous deux aient renié le père, c'est la condition sine qua non pour laisser les bien-aimés vivre leur amour. 
Or, cet amour, nous l'avons vu, ne pourra trouver à s'exprimer qu'en exil, loin du père, même si celui-ci fait sans cesse retour, jusqu'à sa mort. Et même alors, sa disparition n'est pas acquise : la volonté de toute-puissance de James est rendue manifeste par sa dernière friponnerie, puisqu'il se pose sur la fin, lui, l'égoïste scélérat, en victime faisant acte de pardon. La posture est éminemment christique, et vise à instaurer sa domination par-delà la tombe. La supercherie est vite éventée par David, mais la mégalomanie saturnienne de James n'en est pas moins étouffante. Il est la plus asphyxiante des figures paternelles, dont l'omniprésence, et la prétention à l'omnipotence, sont le moteur de ce besoin d'exil que ressent David. Contre une société dystopique, car fondée sur l'ubiquité paternelle, le remède que trouve David est de s'expatrier ; contre son gré d'abord, lorsqu'il s'échoue sur l'île d'Earraid dans Kidnapped, puis volontairement, quand il émigre en Hollande, avec le but affiché de devenir Advocate, et donc, symboliquement, de prendre la place de Prestongrange, dont c'est le métier. Le chemin vers l'indépendance du jeune M. Balfour est ainsi parsemé de ces endroits étrangers, étriqués et dont pourtant on n'a jamais complètement fait le tour, des lieux comme en dehors du temps, où domine un sentiment de suspension de l'action. C'est lors de ces pauses, confronté à une solitude toujours mal vécue mais salvatrice, que David trouve à se ressourcer.

10 L'insularité est ainsi pour David la condition d'un contrôle retrouvé sur son destin. Les îles, en effet, ne manquent pas dans le diptyque, que ce soit dans l'un des passages les plus marquants du premier opus (sur l'île d'Earraid) ou, par deux fois, dans sa suite (sur l'île de Bass, sous la garde rapprochée des hommes de Prestongrange, et près de Dunkerque, dans cet endroit retiré qu'est «l'auberge à Bazin» comme l'appelle le guide, où se conclut l'action). Sur Earraid, David apprendra que les obstacles n'en sont pas vraiment (l'endroit n'est une île qu'à marée haute), et qu'il a les ressources et la volonté nécessaires pour survivre à n'importe quelle épreuve; sur Bass, qu'il peut manipuler les édits de ses ennemis (il intrigue pour arriver à ses fins, mais respecte scrupuleusement la lettre, sinon l'esprit, des ordres de Prestongrange) ; en France, dans un passage étonnant de légèreté pour une fin de récit d'aventures, qu'une fuite éperdue suffit pour se débarrasser de ses poursuivants. L'île est un espace dont la caractéristique principale n'est pas tant d'être sauvage que d'être neutre, car aucune autorité ne s'exerce a priori en ce lieu retiré ; libre à David, dès lors, d'y fonder la sienne, par sa ressource et son énergie. C'est en définitive ce qu'il fait sur chacune des îles, où il finit par triompher de ses ennemis et de ses propres failles. Mais peut-être reste-t-il une dernière " île » à explorer, qui ne figure sur aucune carte, et qui se révèle à nous à la faveur d'une conclusion lapidaire qui pourtant modifie tout le sens du récit. On apprend ainsi, à la toute fin de Catriona, sur les deux derniers paragraphes, que les destinataires du récit qui a précédé sont en fait les propres enfants de David et Catriona ; précision tardive, sur laquelle le lecteur court le risque de ne pas s'arrêter, mais qui change considérablement la donne.

11 Voilà donc M. Balfour en position de père, non pas à la toute fin, mais depuis le tout début. Un brouillage générique se trouve alors introduit dans le texte lui-même, puisque du statut de Bildungs-roman ou de faux Mémoires, il passe, si l'on adopte le point de vue des enfants (destinataires explicites du texte et donc images du lecteur), à celui de bedtime story, voire de roman à clef. On pourra trouver que c'est pousser la suspension d'incrédulité un peu loin, mais cette dernière manœuvre de Stevenson fait bien partie du programme du texte, puisqu'elle vise à poser de manière définitive 
l'autorité de David, comme narrateur et comme père. David, en effet, est le seul à avoir voix au chapitre dans ces deux paragraphes au discours direct sans marqueurs de citation. Point ici d'ouverture au dialogisme; il ne reste à ce stade que l'univocité de son seul discours, qui se veut l'expression d'une autorité bienveillante, voire complice, vis-à-vis de ses enfants, prénommés Alan et Barbara, en souvenir de leurs glorieux aînés, Alan Breck l'insoumis et Barbara Grant la déférente. Cependant, il est (gentiment) défendu aux enfants de juger leurs parents (alors que David leur en a fourni toutes les armes), car ils sont tout aussi déraisonnables qu'eux à l'époque ; David le père n'est pas si prompt qu'il y paraît à laisser son ascendant paternel s'effriter. En outre, il est intéressant de constater qu'en appelant ses enfants du nom des deux personnages qui incarnent les deux extrêmes vis-à-vis de l'autorité dans le système des romans, il les « intègre » en les soumettant in fine à sa loi ; faisant d'une pierre deux coups, il « infantilise » Alan et Barbara, sur lesquels il a enfin autorité, en tant que pater familias, mais aussi le lecteur, qui n'est pas placé plus haut qu'eux, puisqu'ils sont sa figuration dans le texte. Kidnapped et Catriona s'avèrent donc bien être des récits à hauteur d'enfants, mais pas comme une lecture sommaire aurait pu le laisser croire. David, en ces deux derniers paragraphes, se saisit enfin de cette omnipotence paternelle tant convoitée. D'une part, la loi des hommes ne s'applique pas à Shaws, où semble de nouveau établie la Maison des Balfours (on peut, comme il encourage son fils à le faire, y porter un toast au souverainiste Charles Stuart, le « roi de par-delà les eaux » sans encourir les foudres de la justice qui partout ailleurs punirait ce crime de lèse-majesté de mort); d'autre part, il a, au niveau narratif, le dernier mot, en patriarche bienveillant qui sait tirer les leçons de sa vie passée. Cette disposition presque autocratique n'est possible qu'en position de retrait, dans l'espace (Shaws semble retiré du monde) et dans le temps (le bond temporel brutal effectué par les deux derniers paragraphes les isole complètement du reste de l'histoire, et impose un présent en décrochage total du passé révolu du reste du texte). C'est ce que nous appellerons une insularité utopique, associant les sens pleins d'ou-topos, ou «nulle part », et d'eu-topos, ou « lieu de bonheur ».

Les deux derniers paragraphes du diptyque sont donc le siège immatériel d'un Paradis retrouvé, accessible aux seuls Balfour, et où se suspend toute loi autre que celle de David. Il faut cependant bien prendre conscience de ce que cette suspension a d'exceptionnel, contrairement à une tradition persistante qui voudrait qu'elle soit la norme chez Stevenson. Ainsi, pour ne se préoccuper que de ses seuls autres romans écossais, The Master of Ballantrae (1889) et The Weir of Hermiston (inachevé, 1896), on constate que l'auteur s'attache effectivement à poser le monde comme simple théatre d'une lutte fratricide (pour le premier) ou infanticide (pour le second), entre deux adultes cette fois. Mais ce n'est plus une utopie que la longue lutte à mort de deux membres d'une même famille, et l'insularité chez Stevenson a aussi un versant noir, dystopique, celui d'un monde non plus adolescent et relativement léger, mais adulte et véritablement tragique où, si les protagonistes sont obsédés par leur lutte au point de se détourner du monde, leur punition est une destruction réciproque ; et ce n'est pas la moindre des qualités de Stevenson que d'avoir su ménager cette dernière ambiguïté. 


\section{NOTES}

1. Jean-Pierre Naugrette, Robert Louis Stevenson: l'Aventure et son Double, Paris, Presses de l'École normale supérieure, 1987.

2. Robert Louis Stevenson, Kidnapped (1886), Robert Louis Stevenson: The Scottish Novels, Édimbourg, Canongate Classics, 1995.

3. Robert Louis Stevenson, Catriona (1893), Robert Louis Stevenson: The Scottish Novels, Édimbourg, Canongate Classics, 1995.

4. Alan Sandison, Robert Louis Stevenson and the Appearance of Modernism: A Future Feeling, London, Macmillan Press LTD, 1996.

5. Jerome Hamilton Buckley, Season of Youth: The Bildungsroman from Dickens to Golding, London, Harvard University Press, 1974.

6. Op. cit., p. 17-18.

7. Op. cit., p. 19.

8. Walter Scott, Waverley, or,'Tis Sixty Years Since (1814), Oxford, Oxford University Press, 1986.

9. Op. cit., p. 39.

\section{AUTEUR}

\section{CYRIL BESSON}

Université Stendhal-Grenoble 3 